\title{
The design and research of logistics structure system based on computer network
}

\author{
He Jinlian ${ }^{1, a}$ \\ ${ }^{1}$ Jiangxi Science \& Technology Normal University, Nanchang, Jiangxi, 330013, P.R. China \\ ajxsyhjl@163.com
}

Keywords: Logistics, Architecture, Computer network

\begin{abstract}
It is an important content of the logistics enterprises to standardize the management of logistics enterprises. Through the research on the logistics enterprise logistics system, the information management system can be effectively solved.
\end{abstract}

\section{Introduction}

With the application of information technology in logistics enterprise management, it has brought profound changes, which makes the logistics enterprises to form a complete closed-loop management system from production, sale and purchase. However, in the process of logistics management, computer technology will produce a variety of information, and the relationship between the complex and the structure of each system is difficult to coordinate, how to effectively use the computer network to develop a composite structure of logistics management, logistics management information to achieve dynamic synthesis and tracking, has a very important role.

\section{Design and development of logistics system based on computer network}

Through the analysis of the logistics enterprise's demand, we need to construct the specific service content. In the process of these specific services, we need to have a lot of technical components to realize these functions. This is the important content of the computer network. It can realize the service content by using WebServices server, which can effectively integrate the various service functions of the computer network and logistics management system.

\section{Development and deployment of computer network operation platform}

The information structure of the computer network logistics management system is mainly used to construct the distributed management mode, which is integrated with the traditional logistics management system. The system involves many different management platforms, and the platform of Delphi,.Net, J2EE, etc.. These systems are in the promotion of the development of logistics enterprise information, these system architecture to the logistics enterprise's safety, production plays a key role.

\section{Realization of logistics system based on computer network}

Using computer network to carry out the logistics management system, which is based on a certain standard, standardization, flexible and easy to design, it can combine the original system and the network structure of the logistics enterprise, realize the logistics enterprise management and the flexible configuration and management of the business.

Single sign on the logistics management system and centralized authorization. Logistics management system uses a single point of access to the system management, the logistics business management has a special business needs of management, information management system to provide WebService verification interface, the use of database system authentication, users only need to log in through the portal system, users can be different in different architecture system, and do not need to log in again and again: 
Logistics management system of the management or user only through the system management of the home side of a landing, you can realize the landing to the information management system and enterprise information management system, the effective solution to the cross platform operation between the system. In the information system, the main process of the single point landing is as follows:

When the system user needs to log in the original information management system A, A system will jump to the main landing page of the logistics information management system, users enter the user login credentials and the identity token, then in the jump to the original information system architecture, this time, the original information system A will detect the token information and computer network information management system to obtain the user's login credentials, when the token to allow users to access the original information management system A page, but also in the original information management system A users to manage local documents. Second is in the original information management system A to log in to the logistics enterprise information system A, because the landing information management system $\mathrm{A}$ has completed the acquisition of the token, the system B will use this token in the computer logistics management system to obtain the user credentials, when the system B from the logistics management system to obtain the user credentials, you can allow users to log in to the original information management system $B$, while retaining the original user credentials in the system B, can effectively build the user's access to the network.

Logistics information management system provides the authorization management including system level access control and multi granularity access control two management functions. The system will work personnel positioning management, logistics and management system, attendance information system related business upload to the FTP server, so the attendance management system will download the corresponding attendance management information and analysis, and finally to the XML system to the performance of the staff, and the information is uploaded and stored in the attendance information management system database, and then through the periodic call task file, and then generate staff attendance information management file.

Function development of logistics system bus management. In the design and development of logistics information management system, for the architecture of the system mainly uses is the idea of hierarchical design to achieve the system's development, architecture system is divided into database, component layer, the logistics bus, service interface layer, attendance, orders, several client level, through the logistics service bus for the entire system architecture provides management services platform, to screen out the differences between the various components of the system, to logistics enterprise service each organic straightforward combination in the system of service bus will be effective. In the course of service implementation of the subsystems, the form of the sub system is provided to the service bus, and then the logistics information management system will be based on the form data provided by the service bus. In the design of the interface layer of the computer network logistics information architecture, the interface of WPF is mainly used in the.Net system, which can be easily realized by the presentation layer, or other architecture data.

\section{Design of information management system based on computer network}

The architecture design of the logistics enterprise information management system, mainly uses the dual bus architecture model to design, that is, the service bus and data bus structure model. The system's service bus is responsible for the logistics enterprise's business data, logical data processing, coordination, scheduling and a series of functions, data bus function is to integrate the data of the original information system, and provide a unified data view.

In the information management system, the synergistic effect of logistics service bus and logistics data bus, for data services for collaborative processing, reduces the system architecture of the coupling degree, reduce the data conflict between different subsystem structure, but also reduce the complexity of system data. Logistics system of internal management information is integrated into the logistics service bus and logistics data bus architecture. It mainly includes management sub system of safe production, operation and management subsystem, decision-making and analysis 
subsystem, and the corresponding subsystem includes the next level of information management system, such as the personnel positioning, staff attendance, purchasing management subsystem architecture. In the process of the system architecture design, the system can provide the service of fine granularity and the arrangement process, which is convenient to manage the information in time. Logistics data bus is the main data management platform of logistics service bus. The main function is to provide the logistics data. The workflow engine in the information management system is to realize the management of business process and business data processing, such as examination and approval of the framework:

\section{Conclusion}

Enterprise logistics information management system is based on the original information management system of the logistics enterprises. Through the integration of these information systems, the information system framework can effectively integrate the various information management system. It can realize the integration and management of various information.

\section{References}

[1] Highlights. Research on university data warehouse construction. Journal of Wuhan University (SCIENCE EDITION), 2012,58 (S1): 179-184.

[2] Wang is also the application of data warehouse technology based on business report data research . Beijing: China University of Geosciences (Beijing), 2010

[3] H. Profiles in Performance: Business Intelligence Journeys and the Roadmap for Change. New York: John Wiley and DRESNER Sons, 2009 